\title{
Effects of time-varying relative spectral response on the calibration of MODIS reflective solar bands

\author{
Kevin A. Twedt*a ${ }^{\mathrm{a}}$, Amit Angal ${ }^{\mathrm{a}}$, and Xiaoxiong Xiong ${ }^{\mathrm{b}}$ \\ ${ }^{a}$ Science Systems and Applications Inc., Lanham, MD 20706, USA \\ ${ }^{\mathrm{b}}$ Sciences and Exploration Directorate, NASA/GSFC, Greenbelt, MD 20771, USA
}

\begin{abstract}
Calibration of the on-orbit gain changes of the narrow bandwidth reflective solar bands (RSB) of Terra and Aqua MODIS is usually based on the band center wavelength. The relative spectral response (RSR) of each band is assumed to be constant on orbit and the time dependence of an overall gain factor is calculated. Any on-orbit changes to the RSR of the MODIS bands will introduce some error into the calibration and may also have an impact on the Earth scene radiance retrieval. We consider two different ways to track how the RSR of the MODIS RSB may be changing on orbit, and the effect that these changes will have on the calibration. First, we examine in-band RSR measurements from the spectro-radiometric calibration assembly (SRCA) carried on-board both MODIS instruments. Second, we study the broadband degradation of the MODIS scan mirror and how it may be changing the effective out-of-band response of the RSB. We find that RSR changes have a small effect on the radiance calibrated using the on-board solar diffuser, generally less than $0.5 \%$ in all cases at any time in the missions, with bands 1,8 , and 9 impacted the most.
\end{abstract}

\section{INTRODUCTION}

The MODIS instruments on board the Terra and Aqua spacecraft have 20 reflective solar bands (RSB) with wavelengths spanning $412 \mathrm{~nm}$ to $2130 \mathrm{~nm}$ and bandwidths varying from $10 \mathrm{~nm}$ to $50 \mathrm{~nm} .{ }^{1,2}$ The gains of the MODIS RSB are tracked on orbit using a combination of data from an on-board solar diffuser (SD), regular lunar observations, and pseudo-invariant desert targets. ${ }^{3,4}$ To accurately relate the digital response of the MODIS bands to the observed scene radiance or reflectance, the full spectral response of the instrument as a function of wavelength needs to be considered. The spectral response of both MODIS instruments, including both the in-band (IB) and out-of-band (OOB) components, was measured extensively prior to launch. ${ }^{5}$ Currently, the MODIS Characterization Support Team (MCST) calibrates the on-orbit changes in the RSB gains based on the wavelength-integrated response of each band, i.e. the relative spectral response (RSR) is assumed to be unchanged on-orbit so that the time-dependence of the spectral response of each band can be represented by a simple multiplicative factor that is independent of wavelength. This approach has worked very well due to the relatively narrow bandwidth of the MODIS bands.

However, any changes to the RSR of the bands on-orbit will introduce some error into the calibration and may also have an impact on the Earth scene radiance retrieval. We consider two different ways to track how the RSR of the MODIS RSB may be changing on orbit, and the effect that these changes will have on the calibration. First, we examine measurements from the spectro-radiometric calibration assembly (SRCA) carried on-board both MODIS instruments. The SRCA is a unique calibrator that was designed to track the radiometric and spectral performance of the MODIS RSB on orbit, as well as the spatial performance of all MODIS bands (RSB and thermal emissive bands). The SRCA has provided direct measurements of the IB RSR of most RSB multiple times per year since launch. ${ }^{6-9}$

Second, we consider how the broadband degradation of the MODIS scan mirror may be changing the effective OOB response of the RSB. Since launch, the gains of the RSB have experienced changes, most strongly in the blue wavelengths, primarily due to the optical degradation of the unshielded scan mirror. The wavelength and time dependent optical degradation alters the effective RSR of each of the bands. The magnitude of the effect of this modulated RSR depends on the magnitude and wavelength of the primary OOB components relative to the central band peak. This type of time-dependent modulated RSR is included in the calibration of VIIRS, ${ }^{10}$ a follow-on instrument to MODIS that has a very similar design, and has also recently been considered for calibration of the ocean color bands of Aqua MODIS. ${ }^{11}$

*kevin.twedt@ssaihq.com 
The paper is organized as follows. In section 2, we review the pre-launch measured in-band and out-of-band RSR for the MODIS RSB and consider how the pre-launch RSR affects the SD-based calibration. In Section 3, we present IB RSR measurements from the SRCA spectral calibrations, and calculate the effect of the on-orbit RSR changes on the calibrated reflectance and radiance of each band. In Section 4, we review the degradation of the RSB determined using our current calibration methodology and attempt to separate the optical degradation of the scan mirror from other instrument effects. We then calculate the modulated RSR and the impact on the calibrated radiance. Throughout the paper, we focus on the impact of time-dependent RSR on the SD-based calibration. In Section 5, we summarize the results and provide a brief discussion on the implications for calibration based on lunar and desert sites, and for Earth scene radiance retrieval. We focus primarily on results for the VIS/NIR bands in this paper. The OOB RSR for the SWIR bands (HgCdTe detectors) and the VIS/NIR bands (Si detectors) generally do not overlap, so on-orbit changes in the VIS/NIR wavelength range will not impact the SWIR bands, and vice versa. Also, the SWIR bands have had known issues since launch with large OOB response in the mid-IR wavelength range as well as electronic crosstalk effects ${ }^{12}$ which complicate any on-orbit RSR analysis.

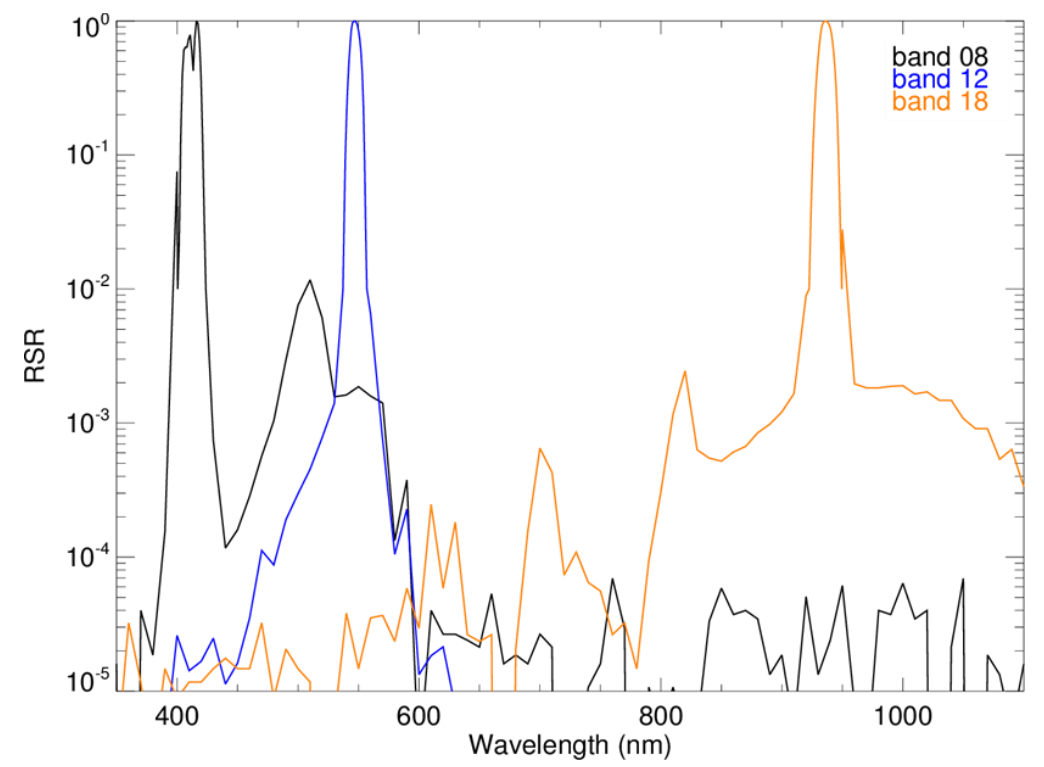

Figure 1. Examples of merged IB and OOB RSRs for Aqua MODIS.

\section{PRE-LAUNCH RSR}

\subsection{Pre-launch RSR measurements}

Both the in-band and out-of-band spectral responses of the MODIS detectors were measured during pre-launch testing. ${ }^{5}$ The MODIS OOB response was characterized using broadband filters and a double monochromater to scan the OOB spectral response of each detector. Figure 1 shows the Aqua MODIS prelaunch RSR (in-band and OOB combined) for some example VIS/NIR bands. The magnitude of the OOB RSR at wavelengths away from the band central wavelength is largest for Aqua MODIS band $8(412 \mathrm{~nm})$, where it exceeds $1 \%$ around $500 \mathrm{~nm}$. For all other bands of both instruments, the peak OOB RSR is less than $0.4 \%$.

The pre-launch measured in-band RSR has been used in the MODIS Level 1B (L1B) radiance product since the beginning of the MODIS missions, where the in-band response is defined as the response around the band center wavelength above $1 \%$ of the peak response. The primary products of the MODIS RSB are the top-of-atmosphere reflectance and radiance, defined as

$$
\begin{gathered}
\rho_{E V} \cos \left(\theta_{E V}\right)=m_{1} d n_{E V}^{*} d_{E S}^{2} / R V S_{E V} \\
L_{E V}=\frac{\rho_{E V} \cos \left(\theta_{E V}\right) \overline{E_{\text {sun }}}}{\pi d_{E S}^{2}}=\frac{m_{1} d n_{E V}^{*} \overline{E_{\text {sun }}}}{\pi R V S_{E V}}
\end{gathered}
$$


where $d n_{E V}^{*}$ is the digital response of each EV pixel corrected for background and instrument temperature variations, $d_{E S}$ is the Earth-Sun distance in astronomical units, $m_{1}$ is a calibration coefficient and $R V S_{E V}$ is the response versus scan angle of the instrument at the angle of the EV pixel. The $\overline{E_{\text {sun }}}$ term is the integral of the solar spectrum over the RSR of each MODIS detector,

$$
\overline{E_{\text {sun }}}=\frac{\int R S R(\lambda) E_{\text {sun }}(\lambda) d \lambda}{\int R S R(\lambda) d \lambda}
$$

In the L1B products, only the in-band RSR is used in calculating $\overline{E_{\text {sun }}}$ for the L1B radiance. The RSR (and $\overline{E_{\text {sun }}}$ ) is considered to be constant in time and has not been updated on orbit. Throughout the rest of this paper, we use the full pre-launch IB/OOB RSR in all calculations, and in Sections 3 and 4 we will allow the RSR and thus the $\overline{E_{\text {sun }}}$ term to vary in time. As a result, the value of $\overline{E_{\text {sun }}}$ and thus the absolute radiance values calculated will be slightly different from the official L1B values even at $t=0$. The relative difference between $\overline{E_{\text {sun }}}$ calculated with only IB RSR compared to the merged IB/OOB RSR is less than $0.25 \%$ for all RSB with the exceptions of Terra band $13(0.6 \%)$, Terra band 14 (0.7\%), Aqua band $8(0.7 \%)$, and Aqua band 15 (0.4\%).

\subsection{Effect of pre-launch RSR on SD calibration}

We first consider the effect of including the pre-launch RSR in the calculation of the SD calibration. The pre-launch IB RSR was used in calculating the pre-launch band-averaged values of the SD bi-directional reflectance factor for each band, but the RSR (pre-launch or on-orbit change) is not currently used in the MCST algorithm for calculating on-orbit changes to the reflectance calibration coefficient $m_{1}$. Even though in this section we consider the RSR to be constant in time, there will still be a time-dependent effect on the SD calibration, due to the changing reflectance (i.e. degradation) of the SD on orbit.

Consider that the retrieved radiance for a given MODIS band is actually the scene radiance integrated over the RSR of the instrument, such that Eq. 2 can be written

$$
L_{E V}(B)=\frac{\int R S R(\lambda, B) L_{\text {scene }}(\lambda) d \lambda}{\int R S R(\lambda, B)}=\frac{m_{1}(B) d n_{E V}^{*}(B) \overline{E_{\text {sun }}(B)}}{\pi R V S_{E V}(B)}
$$

The reflectance calibration coefficient is defined from the solar diffuser calibration as

$$
m_{1}(B)=\frac{\rho_{S D}(B) \cos \left(\theta_{S D}\right)}{d n_{S D}^{*}(B) d_{E S}^{2}} \Gamma_{\mathrm{SD}} \Delta_{\mathrm{SD}}(B)
$$

where $\rho_{S D}$ is the bi-directional reflectance of the solar diffuser measured pre-launch, $\Gamma_{S D}$ is the vignetting function of the solar diffuser screen, $\Delta_{S D}$ is the on-orbit degradation of the SD reflectance, $d n_{S D}^{*}$ is the background and temperature corrected digital response of the SD sector, and $\theta_{S D}$ is the angle between the solar vector and the SD surface. In Eqs. 46 , we use $B$ to denote the terms that are band-dependent and to make the distinction between band dependence and wavelength dependence. Of course, several of the terms also depend on detector, sub-frame, and scan mirror side, which we exclude from the equations for clarity.

Using Eq. 4, we can re-define the calibration coefficient for SD calibration including RSR by considering the scene to be the radiance of the $\mathrm{SD}$, such that

$$
\begin{aligned}
m_{1}(B)=\frac{\cos \left(\theta_{S D}\right) \Gamma_{S D} \int R S R(\lambda, B) \rho_{\mathrm{SD}}(\lambda) \Delta_{S D}(\lambda) E_{\text {Sun }}(\lambda) d \lambda}{d n_{S D}^{*}(B) d_{E S}^{2} \overline{E_{\text {Sun }}}(B) \int R S R(\lambda, B)} \\
=\frac{\cos \left(\theta_{S D}\right) \Gamma_{S D} \rho_{S D}(B) \int R S R(\lambda, B) \Delta_{S D}(\lambda) E_{\text {sun }}(\lambda) d \lambda}{d n_{S D}^{*}(B) d_{E S}^{2} \int R S R(\lambda, B) E_{\text {sun }}(\lambda) d \lambda}
\end{aligned}
$$

The wavelength dependence of $\rho_{S D}$ is very small, so for simplicity we take this term outside the integral and use the band-average $\rho_{S D}(B)$. In the case where there is no wavelength-dependent degradation of the SD reflectance (i.e. $\Delta_{S D}$ does not depend on $\lambda$ ), then Eq. 6 reduces to the usual Eq. 5. However, the SD reflectance degradation does depend on wavelength, and this causes the calibrated gain calculated with Eq. 6 to diverge from the usual calibrated gain calculated with Eq. 5. We calculate these two quantities over the course of the mission for both Terra and Aqua. The ratio of the 
results is presented in Fig. 2. The SD degradation that we use is determined from a time-series fitting of the results from SDSM measurements, which have been extensively described in previous work. ${ }^{13}$

For Aqua MODIS, band 8 shows the largest difference of about $0.5 \%$, whereas all other bands are about $0.1 \%$ or less. It is not surprising that the impact is largest for band $8(412 \mathrm{~nm})$ as the SD degradation is greatest at the lower wavelengths and band 8 has a relatively large OOB RSR. For Terra MODIS, band 8 also sees the largest on-orbit effect of about $0.6 \%$, and the rest of the VISNIR bands in general show a larger effect of the RSR compared to Aqua MODIS. Terra MODIS is more significantly affected mostly because the on-orbit degradation of the SD has been more significant. ${ }^{13}$ These results show the changes that would result from including the pre-launch RSR with no on-orbit updates in the calculation of the calibrated reflectance from the SD calibration. The calibrated radiance would also change if we were to use the full IB/OOB pre-launch RSR: The new radiance would have a time-dependent difference with the official radiance that follows the time-dependence of $m_{1}$ and it would be offset by a constant amount determined by the different in $\overline{E_{\text {sun }}}$ calculated with IB/OOB vs only IB RSR.

The time-dependence shown in Fig. 2 results from the fact that the radiance of the calibration scene (the SD) is changing in time. There is no analogous effect for calibration done with the Moon or pseudo-invariant desert targets. In the Collection 6/6.1 (C6/6.1) L1B products for Terra bands 1-4, 8-10 and Aqua bands 1-4, 8-9, the long-term on-orbit gain changes are calculated using the Moon and desert targets, rather than the SD, though the SD is still used as the at-launch reference and for tracking short-term gain changes and detector differences. Considering this, the largest effect of excluding RSR in the current $m_{1}$ calibration will be for the Terra NIR bands, which see up to $0.3 \%$ effect.
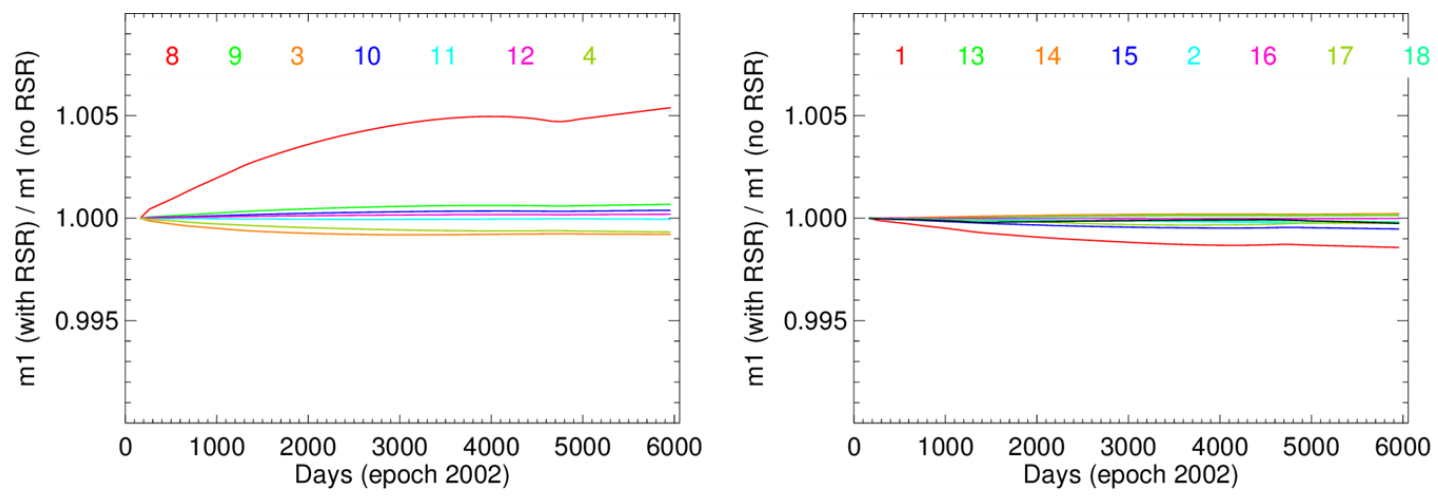

19
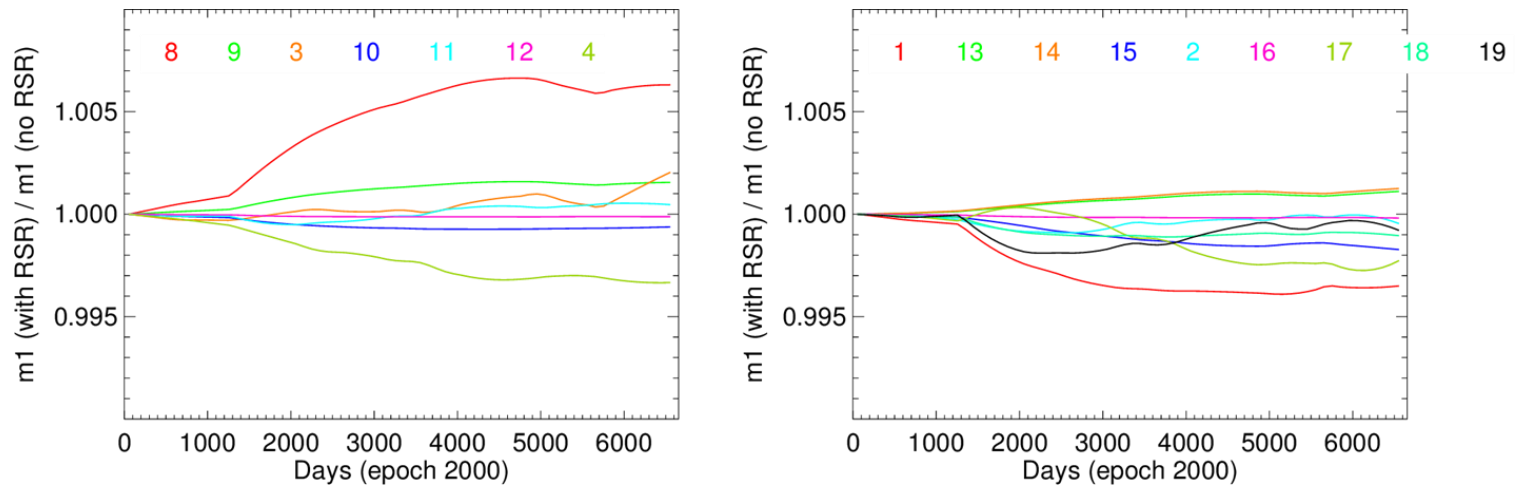

Figure 2. Relative change in $m_{1}$ for (top) Aqua and (bottom) Terra MODIS VIS/NIR bands. For both instruments, the relative change is less than $0.1 \%$ for all of the SWIR bands.

\section{ON-ORBIT CHANGES TO IN-BAND RSR}

We next consider the time-dependent on-orbit changes in RSR that can be measured on-orbit using the on-board SRCA. Operated in spectral mode, the SRCA is designed to scan over most of the IB wavelength range of each of the MODIS bands and measure the relative spectral response of each detector. Detailed descriptions of the SRCA spectral operation and algorithm have been presented before, ${ }^{7}$ as well as results from on-orbit calibrations. ${ }^{8,9}$ The SRCA spectral mode has 
been operated multiple times per year over the course of both Aqua and Terra MODIS missions, providing a significant amount of data to track RSR changes.

Figure 3 shows examples of the IB RSR change as measured by the SRCA at select times in the mission for select bands of Aqua and Terra MODIS. The measurements shown are averaged over all detectors within a band. For Aqua MODIS, all of the bands with acceptable data quality show no significant change in the shape of the in-band RSR with time. For Terra MODIS, several of the bands show significant on-orbit changes in the shape of the in-band RSR, including bands 1, 17, and 19 shown in Fig. 3. Note that for both instruments we do not consider the SRCA spectral results of bands 2, 3, 8 , or 9 in any of this analysis. The accuracy of the results for band 2 are questionable due to a change in measurement configuration from pre-launch to on-orbit. ${ }^{6}$ The results for bands 3,8, and 9 are reliable for early mission calibrations, but due to SRCA lamp failures and scan mirror degradation, the signal-to-noise ratio of the SRCA spectral results is too low to provide reliable results throughout the missions. On-orbit changes in the polarization sensitivity of the scan mirror may also be a complicating factor for bands 3,8 , and 9 .
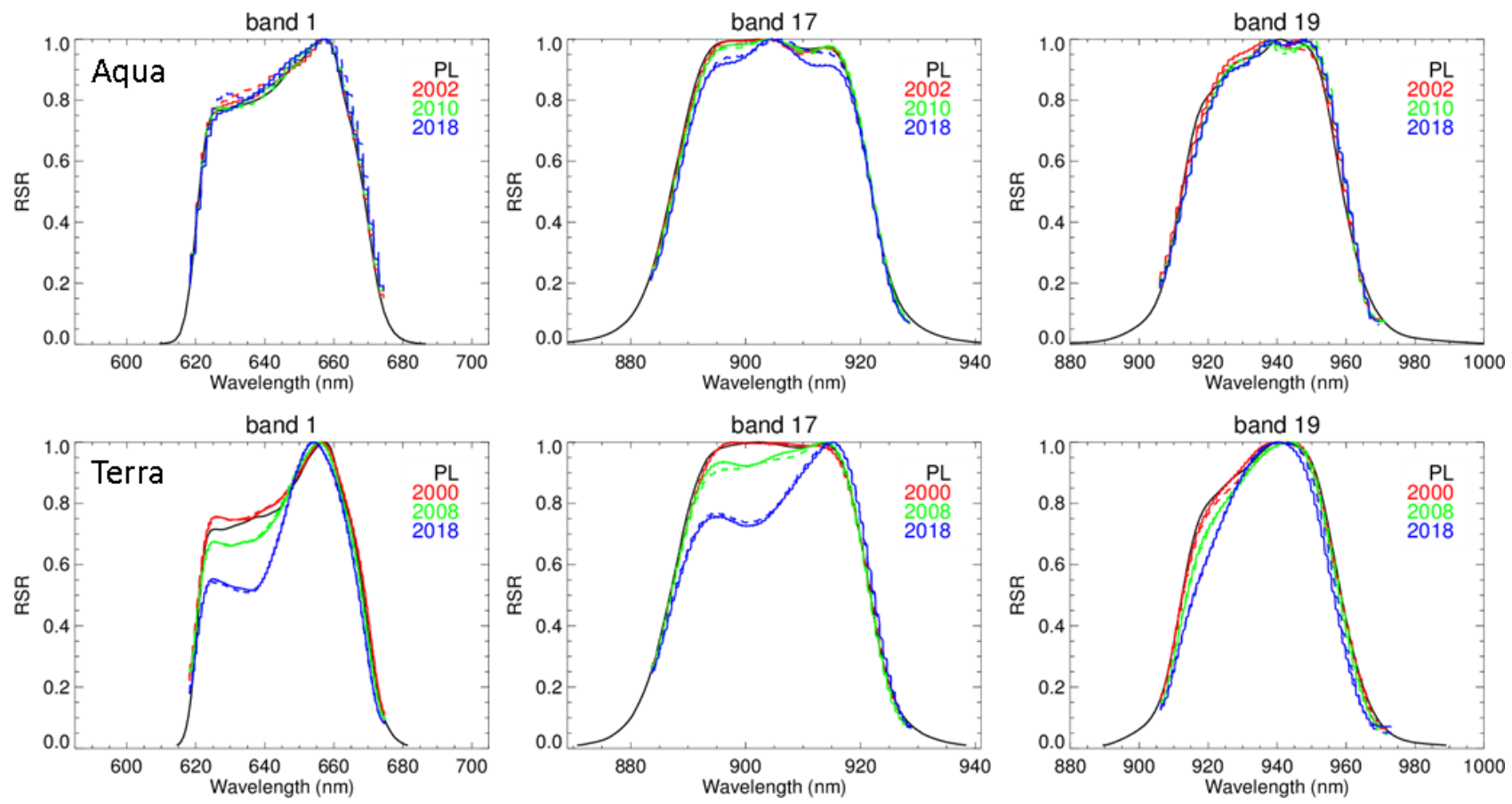

Figure 3. Examples of in-band RSR for select bands of Aqua (top row) and Terra (bottom row) MODIS at select times (years) in the mission. The solid lines represent mirror side 1 results and the dashed lines represent mirror side 2 results. The pre-launch data are from measurements made with a separate spectral measurement assembly (SpMA).

To quantify the in-band RSR changes shown in Fig. 3, we consider the effect of these RSR changes on the on-orbit trends of the calibrated reflectance and radiance. Since the SRCA only tracks the on-orbit changes in RSR over the inband part of the spectra, we merge the SRCA results with the pre-launch RSR measurements. We do this by simply replacing the pre-launch RSR measurement with the SRCA measurement over the valid SRCA measurement range for each SRCA spectral mode calibration, i.e.

$$
R S R(\lambda, t)= \begin{cases}R S R_{\text {pre-launch }}(\lambda) & \lambda \text { outside SRCA measurement range } \\ R_{S R} R_{S R A}(\lambda, t) & \lambda \text { in SRCA measurement range }\end{cases}
$$

By merging the in-band SRCA spectral results with the pre-launch OOB spectral results, we are making the implicit assumption that the maximum optical transmittance is unchanged relative to the magnitude of the OOB spectrum, which is not necessarily true. But the SRCA spectral mode is only designed to measure the relative spectral response, not absolute.

Using this time-dependent RSR from Eq. 7, we can calculate the $m_{1}$ using Eq. 6. Comparing this to our calculation in the previous section of $m_{1}$ using only the pre-launch RSR gives the effect of the time-dependent in-band RSR on the calibrated reflectance: 


$$
\frac{m_{1}(B, t, R S R(t))}{m_{1}(B, t, R S R(0))}=\frac{\int R S R(\lambda, B, t) \Delta_{S D}(\lambda, t) E_{\text {sun }}(\lambda) d \lambda / \int R S R(\lambda, B, t) E_{\text {sun }}(\lambda) d \lambda}{\int R S R(\lambda, B, 0) \Delta_{S D}(\lambda, t) E_{\text {sun }}(\lambda) d \lambda / \int R S R(\lambda, B, 0) E_{\text {sun }}(\lambda) d \lambda}
$$

Similarly, we calculate the effect on the calibrated radiance. In this case, we consider the on-orbit change in the value of $m_{1} \overline{E_{\text {sun }}}$ due to the time-dependent in-band RSR:

$$
\frac{m_{1} \overline{E_{\text {sun }}}(B, t, R S R(t))}{m_{1} \overline{E_{\text {sun }}}(B, t, R S R(0))}=\frac{\int R S R(\lambda, B, t) \Delta_{S D}(\lambda, t) E_{\text {sun }}(\lambda) d \lambda / \int R S R(\lambda, B, t) d \lambda}{\int R S R(\lambda, B, 0) \Delta_{S D}(\lambda, t) E_{\text {sun }}(\lambda) d \lambda / \int R S R(\lambda, B, 0) d \lambda}
$$

For each SRCA spectral calibration, we calculate the ratios in Eq. 8 and Eq. 9. Since the SD degradation and the solar spectrum both change slowly over the wavelength ranges of the narrow MODIS bands, the changing in-band RSR has a minimal effect on either the reflectance or radiance. The results for the effect of in-band RSR changes on the calibrated radiance (Eq. 9) are shown for Terra MODIS in Fig. 4. Band 1 sees the largest effect, up to $0.3 \%$, due to its relatively large bandwidth and relatively large on-orbit RSR change. For Aqua MODIS, the on-orbit trends are less than for Terra and are less than $0.12 \%$ for all bands.
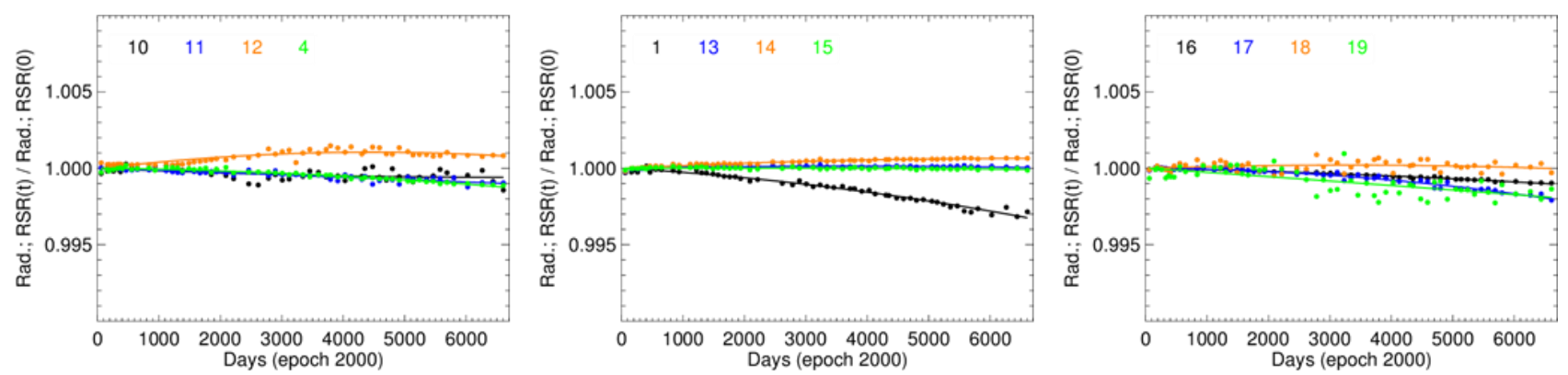

Figure 4. On-orbit impact of IB RSR changes on calibrated radiance for Terra VIS/NIR bands. Each point represents an SRCA spectral calibration and the lines are quadratic fits.

The changes observed by the SRCA for Terra MODIS likely indicate changes to the narrow band filters located in front of the MODIS detectors. Degradation of the scan mirror and other optics is less likely to induce such sharp features. Large changes to the in-band transmission of these filters may very likely be correlated with large changes in the OOB transmission of these filters, which we have no way of tracking on orbit, adding significant uncertainty to attempts to characterize the on-orbit effects of the OOB RSR, which we discuss in Section 4. While the effect of the in-band RSR changes is found to be small for the reflectance and radiance calibration, the effect on the EV products will depend greatly on the spectral properties of the EV scene radiance.

\section{ON-ORBIT CHANGES TO BROADBAND RSR}

\subsection{Aqua MODIS}

We now examine the effect of changes in the broadband optical throughput of the MODIS instruments on the RSR of each band, and the impact on the calibration. Starting with Aqua MODIS, we consider how the calibrated gain of the instrument has changed on-orbit. Figure 5a shows the calibrated gain, $G_{t o t a l}=m_{1}(t=0) / m_{1}(t)$, as a function of band center wavelength for Aqua MODIS at select times in the mission. The $m_{1}$ values in Fig. 5a are from the official C6.1 L1B product. Clearly, Aqua has experienced significantly more degradation in the visible wavelength range compared to the NIR.

The MODIS scan mirror is the primary unshielded optic for MODIS and it is assumed that the majority of the instrument degradation is due to degradation of the scan mirror. This is further evidenced by a clear gain difference between the two sides of the scan mirror, and a clear gain dependence on the angle of incidence of the light on the scan mirror (the RVS). Under the assumption that the gain changes are dominated by changes to the scan mirror (and other optical elements that are in the portion of the optical path shared by all bands), then all bands will experience a broadband optical degradation. This broadband degradation can be applied to the pre-launch measured OOB RSR of each detector in order to provide an on-orbit update to the OOB RSR. 

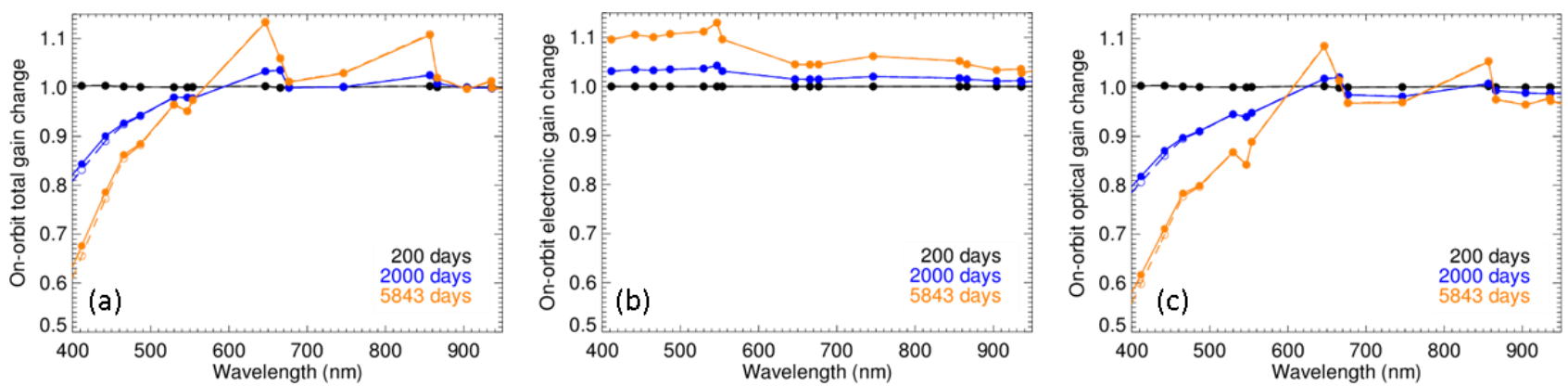

Figure 5. (a) On-orbit gain change of Aqua MODIS VIS/NIR bands at select times during the mission (days since Jan 1, 2002). Both mirror side 1 (solid line) and mirror side 2 (dashed line) results are shown. (b) On-orbit electronic gain change from E-cal measurements. (c) On-orbit optical gain change.

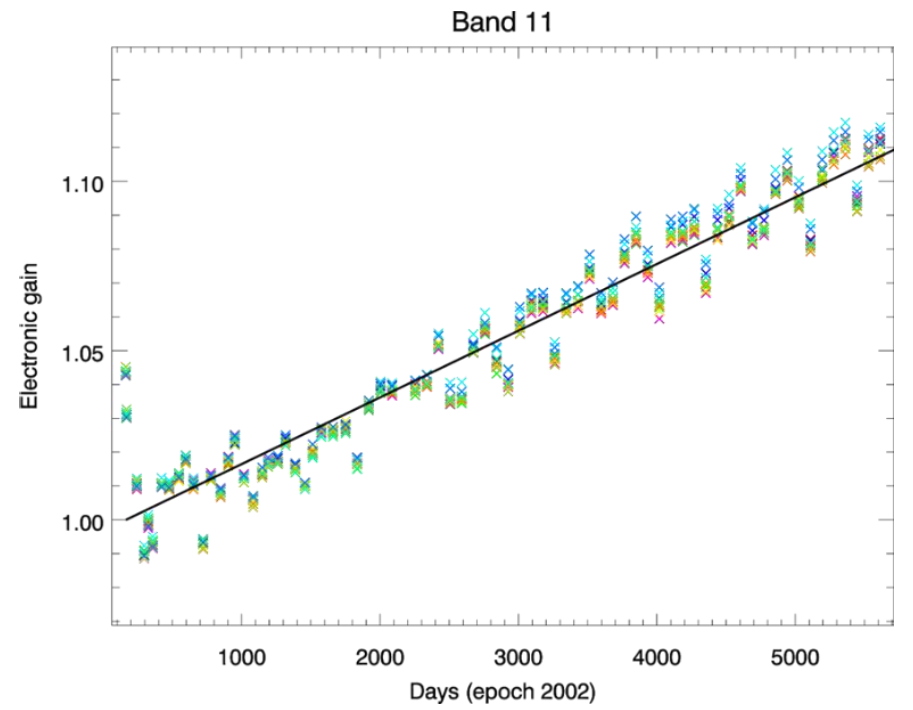

Figure 6. On-orbit electronic gain change of Aqua MODIS band 11. For each calibration time, different colored symbols indicate the results of different detectors, showing the spread in the results over all detectors in the band.

But for this approach to work, we must first isolate the degradation of the scan mirror from that of other optical and electronic components that may experience on-orbit changes and contribute to the gain degradation seen in Fig. 5a. We can consider the total gain change to be composed of changes in electronic gain, $G_{\text {elec }}$, detector gain, $G_{d e t}$, reflectance of the scan mirror and primary optics, $\rho_{\text {primary }}$, reflectance of dichroic elements and focusing optics specific to each focal plane assembly (FPA), $\rho_{\text {fpa }}$, and the transmittance of the narrow band filters, $T_{\text {filter }}$ :

$$
G_{\text {total }}(B, D, S, M) \propto G_{\text {elec }}(B, D, S) \int G_{\text {det }}(\lambda, B, D, S) \rho_{\text {primary }}(\lambda, M) \rho_{f p a}(\lambda, F P A) T_{\text {filter }}(\lambda, B, D) d \lambda
$$

where we have specified the dependence of each term on the band (B), detector (D), sub-frame (S), and mirror side (M), and it is assumed that all quantities can change with time.

Fortunately, the MODIS instruments are equipped with the capability to monitor the changes in $G_{\text {elec }}$ through on-orbit electronic calibrations (E-cals) that have been performed on a regular basis for both instruments since launch. During an E-cal, the MODIS detectors are detached and replaced by a voltage ramp that is applied during the time of space view sector data collection. The change in measured $\mathrm{dn}$ in response to the voltage ramp is used to calculate the electronic gain, and monitor changes in this gain on orbit. More details on the MODIS E-cals can be found in previous publications. $^{14}$

Figure 6 shows the $G_{\text {elec }}$ for an example band over the course of the Aqua mission. For all Aqua and Terra MODIS bands, the $G_{\text {elec }}$ is found to increase gradually over the mission. For each band, we fit the on-orbit electronic gain change (averaged over all detectors within a band) to a polynomial function of time to derive smooth trends. Figure $5 \mathrm{~b}$ 
shows the electronic gain change as a function of band wavelength at select times in the mission. Generally, the VIS bands and the NIR bands tend to have similar changes in electronic gain, which is not surprising since each set of bands uses its own shared ADC. Considering only the average values over all detectors and sub-frames within a band, we can write the optical gain of the instrument as

$$
G_{\text {optical }}(B, M, t)=\frac{G_{\text {total }}(B, M, t)}{G_{\text {elec }}(B, t)}
$$

Figure $5 \mathrm{c}$ shows the calculated values of $G_{\text {optical }}$ as a function of wavelength. If this optical gain change were due entirely to degradation of the scan mirror, our expectation is that this would be a smooth function of wavelength, without any sharp features in the spectrum. This is mostly true in Fig. 5c, but notably bands 1 and 2 have considerably higher gain and still see an increase in $G_{\text {optical }}$ on orbit. As it is quite unlikely that the optical throughput of the instrument has increased at any wavelength on orbit, the on-orbit trends in the gains of bands 1 and 2 likely come from a systematic effect not yet identified.

The $G_{\text {optical }}$ in Fig. 5c still includes the integrated effects of the detector gain and filter transmittance, as well as the reflectivity of the optics. All of the VIS/NIR bands use Si detectors that likely degrade in a similar way. It is known that these detectors experience gain degradation under radiation exposure initially in the NIR wavelengths. The relatively stable on-orbit performance of the NIR bands (e.g. 17-19) for Aqua MODIS indicates that the Si detectors have not experienced any significant radiation damage on orbit, thus it is likely that $G_{\text {det }}$ is fairly stable on orbit for all VIS/NIR bands. The SRCA spectral results, which show no significant change in the in-band RSR of the Aqua MODIS bands, provide a good indication that the band filters have not experienced significant degradation.

It is likely that, with the exception of bands 1 and 2, the optical degradation shown in Fig. $5 \mathrm{c}$ is dominated by the degradation of the scan mirror and this wavelength-dependent degradation can be applied to the relative spectral response of all bands equally. We define the on-orbit modulated RSR to be

$$
R S R(\lambda, B, t)=\frac{R S R(\lambda, B, 0) G_{\text {optical }}(\lambda, t)}{\max \left[R S R(\lambda, B, 0) G_{\text {optical }}(\lambda, t)\right]}
$$

where $G_{\text {optical }}$ is interpolated between bands to get a continuous function of wavelength.

Similar to our analysis in Section 3, we determine the effect of the changing on-orbit RSR on the calibrated reflectance and calibrated radiance (of the SD calibration). We calculate the ratio in Eq. 9 using the RSR from Eq. 12 and plot the results in Fig. 7. The on-orbit impact is up to $0.4 \%$ for band 8, up to $0.1 \%$ for band 9 , and negligible for all other bands.
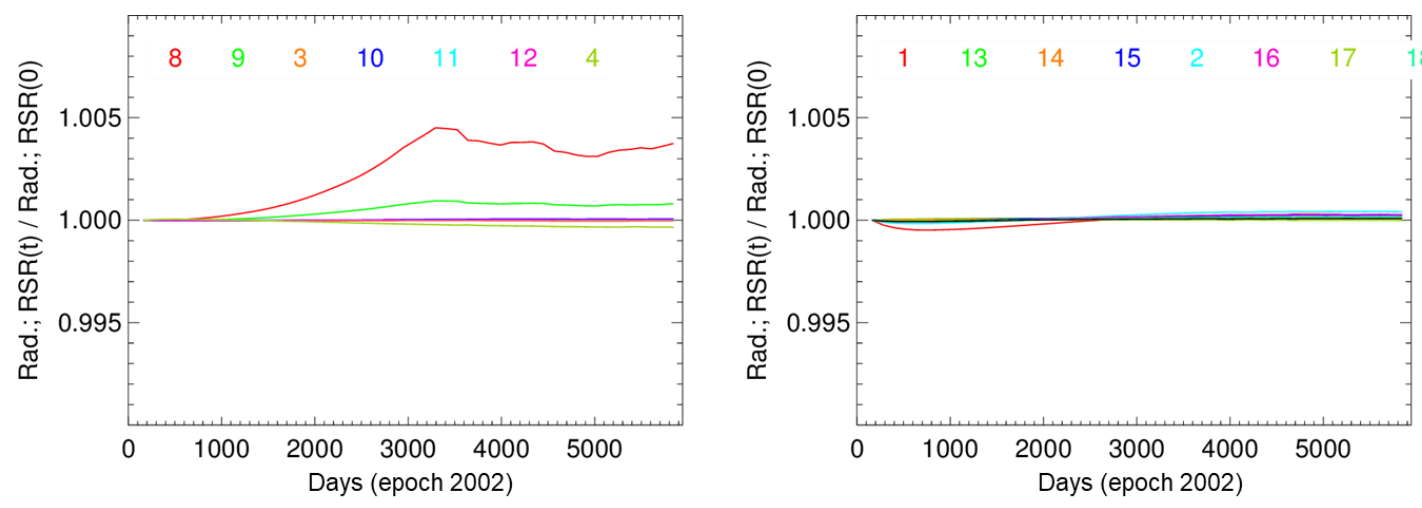

19

Figure 7. On-orbit impact of broadband RSR changes on calibrated radiance for Aqua VIS/NIR bands.

A similar analysis on OOB RSR impact was recently published for Aqua MODIS ocean color bands, ${ }^{11}$ and we generally find agreement with those results.

\subsection{Terra MODIS}

Following the example of Aqua MODIS, we now present the results for Terra MODIS. Figure 8a shows the on-orbit gain change of the Terra VIS/NIR bands as a function of wavelength for select times during the mission. Figure $8 \mathrm{~b}$ 
shows the on-orbit change in electronic gain, and Fig. 8c shows the on-orbit change in optical gain, following the same analysis as was done for Aqua.

Clearly, the on-orbit degradation of the Terra MODIS instrument as a function of wavelength carries significantly more features than Aqua MODIS. It is still likely that the degradation of the scan mirror is the primary cause of the drop in gain for Terra RSB on-orbit. But there are several features in Fig. 8c that must have a different explanation, for example the large difference in gain between bands that have a similar wavelength, such as bands 11 and 12, or bands 13 and 14 . These sharp differences in gain are more likely to be explained by differences in degradation of the detectors or a drop in the transmittance of the narrow band filters. We calculate a modulated RSR and the effect on the calibrated radiance for Terra in the same way that we did for Aqua, and we find a similar result with band 8 and 9 showing the largest impact with a magnitude of up to $0.5 \%$. But the irregularities in Fig. $8 \mathrm{c}$ place a great deal of uncertainty on the Terra results, and a better understanding of the source of these gain changes is needed to provide a confident analysis.
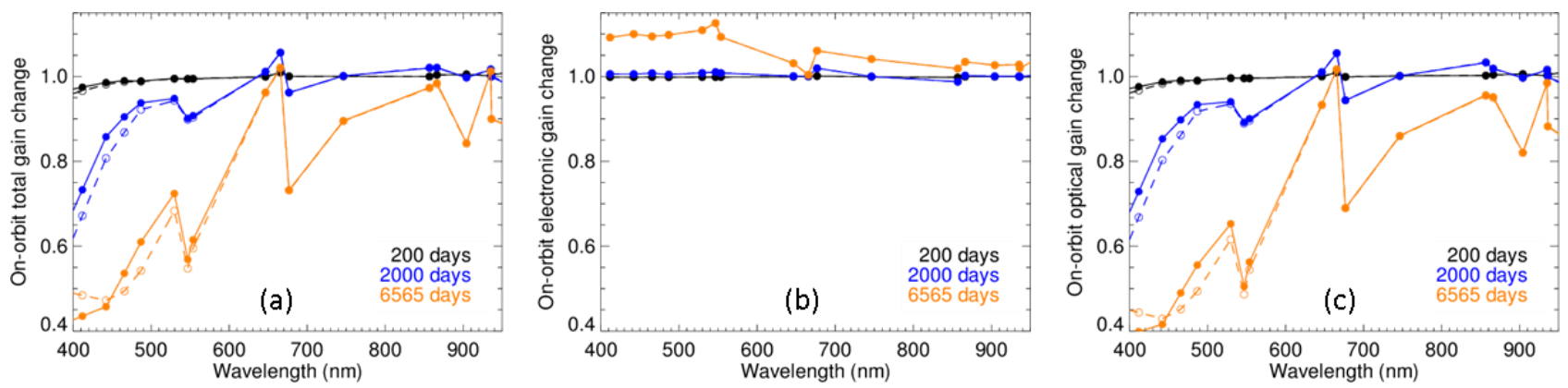

Figure 8. (a) On-orbit gain change of Terra MODIS VIS/NIR bands at select times during the mission (days since Jan 1, 2000). Both mirror side 1 (solid line) and mirror side 2 (dashed line) results are shown. (b) On-orbit electronic gain change from E-cal measurements. (c) On-orbit optical gain change.

\section{CONCLUSION}

We have shown the effect of on-orbit changes in the MODIS VIS/NIR band RSR considering both the in-band RSR changes and the modulated OOB RSR due to broadband instrument degradation. The effect of the RSR changes on the radiance calibrated using the on-board solar diffuser is found to be small, generally less than $0.5 \%$ in all cases at any time in the missions. The effect on reflectance is generally less than the effect on the radiance.

The results in this paper focused on the SD-based calibration and are relevant at the SD angle of incidence of the scan mirror. A similar analysis could be done for lunar-based calibration using lunar spectral models and the instrument gains at the lunar (space view) angle of incidence. Also, pseudo-invariant desert sites are used for calibration at various AOI for select MODIS RSB. A full analysis of the RSR effect on MODIS RSB calibration would involve repeating this analysis for lunar and desert calibration targets and deriving a scan-angle dependent effect. A preliminary analysis indicates that the effect of RSR on lunar- and desert-based calibration will be of the same general magnitude as we found for the SD.

Lastly it is important to note that a changing RSR can have a significant effect on Earth scene radiance retrieval even though the effect on the calibration is small. Earth scenes that have significant spectral features within the bandwidth of one of the MODIS bands could be significantly impacted by modest changes of the in-band RSR of these bands. The large in-band changes seen (Fig. 3) for the Terra water absorption bands 17 and 19 may be of particular interest.

\section{ACKNOWLEDGEMENTS}

We thank Dan Link and Emily Aldoretta for their work on SRCA calibration analysis, and Na Chen for her work on electronic calibration analysis. We also thank Dan Link for his review of this paper. 


\section{REFERENCES}

[1] Barnes, W. L. and Salomonson, V. V., "MODIS: a global imaging spectroradiometer for the Earth Observing System," Proc. SPIE, 10269, 102690G (1992).

[2] Xiong, X., Barnes, W. L., Guenther, B. and Murphy, R. E., "Lessons learned from MODIS,” Adv. Space Res. 32(11), 2107-2112 (2003).

[3] Sun, J., Xiong, X., Angal, A., Chen, H., Wu, A. and Geng, X., "Time-Dependent Response Versus Scan Angle for MODIS Reflective Solar Bands,” IEEE Trans. Geosci. Remote Sens. 52(6), 3159-3174 (2014).

[4] Xiong, X., Wenny, B. N. and Barnes, W., "Overview of NASA Earth Observing Systems Terra and Aqua moderate resolution imaging spectroradiometer instrument calibration algorithms and on-orbit performance," J. Appl. Remote Sens. 3(1), 032501 (2009).

[5] Barnes, W. L., Pagano, T. S. and Salomonson, V. V., "Prelaunch characteristics of the Moderate Resolution Imaging Spectroradiometer (MODIS) on EOS-AM1," IEEE Trans. Geosci. Remote Sens. 36(4), 1088-1100 (1998).

[6] Choi, T., Xiong, X., Wang, Z. and Link, D., "Terra and Aqua MODIS on-orbit spectral characterization for reflective solar bands," Proc. SPIE, 8724, 87240Y (2013).

[7] Montgomery, H., Che, N., Parker, K. and Bowser, J., "The algorithm for MODIS wavelength on-orbit calibration using the SRCA," IEEE Trans. Geosci. Remote Sens. 38(2), 877-884 (2000).

[8] Xiong, X., Che, N. and Barnes, W. L., "Terra MODIS on-orbit spectral characterization and performance," IEEE Trans. Geosci. Remote Sens. 44(8), 2198-2206 (2006).

[9] Link, D., Wang, Z., Twedt, K. A. and Xiong, X. J., "Status of the MODIS spatial and spectral characterization and performance after recent SRCA operational changes," Proc. SPIE 10402, 104022G, SPIE (2017).

[10] Lei, N., Xiong, X. and Guenther, B., "Modeling the Detector Radiometric Gains of the Suomi NPP VIIRS Reflective Solar Bands," IEEE Trans. Geosci. Remote Sens. 53(3), 1565-1573 (2015).

[11] Lee, S. and Meister, G., "MODIS Aqua Optical Throughput Degradation Impact on Relative Spectral Response and Calibration of Ocean Color Products," IEEE Trans. Geosci. Remote Sens. 55(9), 5214-5219 (2017).

[12] Xiong, X., Chiang, K.-F., Adimi, F., Li, W., Yatagai, H. and Barnes, W. L., "MODIS correction algorithm for outof-band response in the short-wave IR bands," Proc. SPIE 5234, 605 (2004).

[13] Chen, H., Xiong, X., Angal, A. and Twedt, K. A., "On-Orbit Characterization of the MODIS SDSM Screen for Solar Diffuser Degradation Estimation,” IEEE Trans. Geosci. Remote Sens. 55(11), 6456-6467 (2017).

[14] Xiong, X., Chen, N., Li, Y. and Wilson, T., "Assessments and applications of Terra and Aqua MODIS on-orbit electronic calibration,” Proc. SPIE, 9972, 99720X (2016). 